\title{
Globe
}

Revue internationale d'études québécoises

\section{Yvan Lamonde et Didier Poton [éd.] : La Capricieuse (1855) : poupe et proue. Les relations France-Québec (1760-1914), Québec, Presses de l’Université Laval, 2006}

\section{Sylvain Simard}

Volume 10, numéro 1, 2007

URI : https://id.erudit.org/iderudit/1000085ar

DOI : https://doi.org/10.7202/1000085ar

Aller au sommaire du numéro

Éditeur(s)

Globe, Revue internationale d'études québécoises

ISSN

1481-5869 (imprimé)

1923-8231 (numérique)

Découvrir la revue

Citer ce compte rendu

Simard, S. (2007). Compte rendu de [Yvan Lamonde et Didier Poton [éd.] : La Capricieuse (1855) : poupe et proue. Les relations France-Québec (1760-1914),

Québec, Presses de l'Université Laval, 2006]. Globe, 10(1), 171-180.

https://doi.org/10.7202/1000085ar d'utilisation que vous pouvez consulter en ligne.

https://apropos.erudit.org/fr/usagers/politique-dutilisation/ 


\section{RECENSIONS}

Yvan Lamonde et Didier Poton [éd.]

La Capricieuse (1855): poupe et proue.

Les relations France-Québec (1760-1914)

Québec, Presses de l'Université Laval, 2006.

Publié sous la direction de Yvan Lamonde et Didier Poton, l'ouvrage réunit les principales interventions présentées dans le cadre du colloque organisé par la Commission franco-québécoise sur les lieux de mémoires communs à l'occasion du $150^{\circ}$ anniversaire du passage de $\mathrm{La}$ Capricieuse, qui fut le premier navire portant pavillon français à remonter officiellement le Saint-Laurent depuis la Conquête anglaise. D'importance et de valeur inégales, ces textes contribuent, chacun à leur façon, à mieux faire connaître un épisode fondateur de l'identité québécoise dans sa relation à la France, telle qu'elle se poursuit, se recrée et se révèle à elle-même tout au long du XIX siècle.

Ce qui sous-tend ces diverses approches, c'est bien le constat que, quelle que soit la différence de perception de cet événement par les historiographies française et québécoise, elles n'en sont pas moins toutes deux légitimes et complémentaires. Tout a été dit depuis les recherches de Jacques Portes, - et certains articles viennent ici en renforcer la pertinence -, sur la faiblesse des relations commerciales et économiques entre la France et le Canada et sur l'extrême circonspection des autorités françaises à renouer quelque lien que ce soit avec son ancienne colonie et à soutenir toute initiative susceptible de susciter la moindre irritation chez les autorités britanniques. La cause est donc entendue, la mission de La Capricieuse est bien le résultat d'une initiative personnelle de P.-H. Belvèze, commandant de la station navale de Terre-Neuve. Elle fut approuvée non sans réticence par le ministère des Affaires étrangères, pour des motifs exclusivement commerciaux et, pour reprendre la formule de Portes, elle «n'appartient pas 
au champ des relations internationales." Mais comme il arrive souvent en histoire, la valeur symbolique et mythique d'un événement nous apprend parfois davantage que l'énumération rationnelle de ses causes et de ses conséquences. Tout ce livre plaide en faveur de la légitimité d'une historiographie autre, celle de la perception comme des attentes, souvent insatisfaites et déçues, suscitées par l'événement dont la mémoire participera aussi à la constitution d'une identité nationale en quête de reconnaissance.

Une première série de textes apporte des points de vue généraux sur l'amont de l'événement éponyme ou sur l'ensemble du siècle, confirmant les thèses de Claude Galarneau qui voit une certaine continuité dans les relations entre le Bas-Canada et son ancienne "mère-patrie». Mais les conclusions varient selon que l'observateur s'élève et tente d'embrasser le long terme ou qu'il se concentre sur un épisode, un groupe, un réseau, une époque précise. Ainsi l'excellent tableau des relations commerciales entre la France et l'Amérique du Nord présenté par Bruno Marnot traduit bien, à l'aide de graphiques très explicites, non seulement le faible volume de ces échanges mais compare, autant que faire se peut, en valeur et en volume les échanges entre le Canada et la France à ceux existant entre les États-Unis et la France. Statistiques nécessairement imparfaites, puisque de 1831 à 1844 ,

la valeur des échanges canadiens est incluse dans le total des valeurs des "possessions anglaises" et qu'à partir de 1845, le Canada appartient à la rubrique "Antilles anglaises"; on gagne en précision en 1869 avec la mention "Antilles anglaises du Nord" [sic] qui devient "possessions anglaises du Nord" en $1870[\ldots]$; puis le Canada [étant...] individualisé à partir de $1895^{1}$.

Quoi qu'il en soit, les conclusions sont claires et démontrent bien qu'en dépit d'efforts isolés, on constate une faiblesse chronique des échanges, tout au long du siècle, qui tranche avec la vitalité, même si elle connaît d'importantes variations, des échanges franco-américains. Le désarmement douanier marqué en 1846 par l'abolition des "Corn Laws" et l'abrogation des "Actes de Navigation" en 1852, donne lieu à certaines initiatives consulaires françaises. De son côté, le Canada présentera sa production aux deux premières expositions universelles de 1855 et 1867 . C'est d'ailleurs dans ce contexte libre-échangiste que s'inscrit l'initiative de Belvèze et l'envoi

$$
\div+\div
$$

1. Bruno MARNOT, "Les relations commerciales entre la France et l'Amértque du Nord au XIX" sièclew, p. 5. Yvan LAMONDE et Didıer POTON [éd.], La Capricieuse (1855): poupe et proue Les relations France-Quebec (1760-1914), Québec, Presses de l'Université Laval, 2006. 
du premier bâtiment militaire français depuis 1760 , initiative appuyée en France par Rouher et son ministère du Commerce et, à Montréal, par divers appels de la Chambre de commerce. Mais en l'absence d'échanges commerciaux directs entre les deux pays ${ }^{2}$ et dans le contexte d'intégration économique de l'ensemble canado-britannique, le Canada reste, pour la plupart des milieux politiques et économiques français, ces "quelques arpents de neige" où le négoce avait plus à perdre qu'à gagner. Nulle part plus qu'à La Rochelle est-on nostalgique de la grande époque coloniale, du commerce maritime avec l'Amérique du Nord et le Canada; mais, comme le reconnaît Didier Poton, malgré certaines initiatives de grands armateurs rochelais, les relations de la Rochelle avec le Canada sont, en 1820, lettre morte et toutes les tentatives, notamment celles visant à reprendre le commerce des fourrures, échouent. Les souvenirs de la grandeur passée ne sauraient être des instruments efficaces pour faire face à la compétition économique qui s'instaure entre l'Europe et l'Amérique du Nord.

Mais l'atonie des échanges économiques ne signifie pas l'absence de tout lien, bien au contraire. Anthony Grolleau-Fricard met en lumière, à l'aide de trois récits de voyage, la mise en place et le développement de l'un des réseaux les plus actifs des relations France-Canada. Ce réseau, qui remonte aux années 1810 , a pour origine l'ouverture à Montréal d'une librairie par Hector Bossange, fils du grand libraire parisien Martin Bossange, et son association avec le libraire-patriote Édouard-Raymond Fabre, laquelle ne s'éteindra vraiment qu'en 1910, au décès du fils de ce dernier, Hecror Fabre, alors représentant du Canada à Paris.

Ces récits de voyage, celui d'Édouard-Raymond Fabre en visite chez son beau-frère (qui a épousé sa sœur Julie) et ami Hector Bossange en mai et juin 1843, celui de son fils Charles-Édouard qui l'accompagne et celui de Louis-Hyppolyte Lafontaine qui s'arrête à Paris de février à mai 1838, nous plongent au cœur d'un réseau politique, familial et économique dont les correspondants canadiens sont Louis-Joseph Papineau et EdouardRaymond Fabre qui canalisent vers Hector Bossange le flot de leurs compatriotes venant vivre à Paris ou visiter la ville. Ces récits dressent le portrait de la très active vie bourgeoise d'une famille prospère, liée notamment au banquier Lafitte et dont les relations politiques, sociales et économiques ne se limitent pas à la France mais se ramifient sur plusieurs

$$
++
$$

2. Si l'on excepte l'initiative sans lendemain de 1883 d'une liaison directe Le Havre- Quebec, il faudra attendre 1913 pour que la Compagnie Générale Transatlantique retente l'expérience. 
continents. Les Bossange ont développé des liens politiques et personnels avec Louis-Joseph Papineau et sont très près du mouvement patriote dont Lafontaine et Fabre furent deux piliers lors de la rébellion de 1837. Ils accueillent et fréquentent volontiers les étudiants, prêtres, commerçants et touristes canadiens de passage ou en séjour à Paris.

Ces récits de voyage offrent donc un témoignage très vivant de la diversité des liens personnels qui existent entre le Bas-Canada et son ancienne métropole. Si les relations Bossange-Fabre étaient déjà bien connues, notamment à travers les travaux de Jean-Louis Roy ${ }^{3}$, le réalisateur François Labonté n'en jette pas moins un nouvel éclairage sur les Bossange en s'intéressant à la correspondance de la famille Papineau où se lit la profondeur des liens qui unissent les deux hommes, surtout à l'occasion des événements entourant la rébellion de 1837 et le séjour de Papineau à Paris de 1839 à 1845.

C'est aussi l'exil de Papineau qu'analyse Yvan Lamonde en le situant autant dans l'ensemble des relations France-Bas-Canada depuis la chute de l'Empire que dans la dynamique interne du milieu patriote. Tout comme Françoise Lejeune, il fait remonter une certaine redécouverte du Canada à la Rébellion et aux réactions suscitées par celle-ci au sein du parti républicain, notamment dans le National, où les journalistes radicaux prennent rapidement fait et cause pour "l'esprit français», c'est-à-dire l'esprit révolutionnaire du "parti français" qui tenait tête aux Anglais ${ }^{4}$. À travers les nombreux articles qu'ils consacrent aux rebellions canadiennes, ils mènent aussi leur propre campagne contre le gouvernement français jugé « trop soumis aux Anglais. " À une époque où les milieux républicains français s'enflamment pour le principe des nationalités, lancent des mouvements d'appui aux causes polonaises ou hellènes, il n'est pas insignifiant de voir les journalistes du National évoquer une population qui, selon les mots d'un de leurs rédacteurs, a gardé "notre caractère", "nos cœurs", "notre langue». Aussi ne faut-il pas s'étonner qu'en éditorial le même journal évoquant "StCharles, St-Denis, bourgs dont les noms nous rappellent nos campagnes, [...] ensanglantés, brûlés par les Anglais", conclut que "jamais aucun peuple ne mérita davantage nos sympathies ${ }^{5}$."

$$
+4+
$$

3. Jean-Louis RoY, Edouard-Raymond Fabre, Libraire et patroue canadien Moncréal, Hurtubise HMH, 1974, p. 46.

4. Françoise LEJEUNE, «Les relarions France-Bas-Canada entre 1837 et 1855 . Le Canada reconquis par la France, la France reconquise par le Canada? ", p. 106. Yvan LAMONDE et Didier POTON [éd.], La Capricteuse (1855): poupe et proue. Les relations France-Quebec (1760-1914), Québec, Presses de l'Université Laval, 2006.

5. Le National, éditorial du 17 janvier 1838. 
Et c'est encore ce même National qui assurera, en mars 1839, le premier accueil fait à Papineau en France. En plus du passionnant récit des vaines tentatives du leader patriote pour infléchir la politique française en faveur du mouvement insurrectionnel canadien comme de la remise en question de son rôle et de son leadership par les Patriotes les plus radicaux, Yvan Lamonde affirme avec raison qu'en dépit de cette campagne agressive de la presse républicaine,

la cause canadienne n'avait pas alors de résonances universelles ou internationales. [...] Vue d'Europe, la crise bas-canadienne n'avait pas trouvé de relais pour s'inscrire dans le réveil des nationalités de 1830 . Les deux pays qui auraient pu y contribuer avaient des raisons de ne pas le faire: la France, désintéressée de son ancienne colonie et alliée à l'Angleterre qui, elle, avait tout intérêt à faire l'impasse sur cette colonie lointaine ${ }^{6}$.

Mais les Bas-Canadiens ne désespèrent pas d'intéresser la France et les Français à leur sort. La publication d'un certain nombre d'ouvrages sur le Canada et de récits de voyages en France, la diffusion beaucoup plus systématique d'ouvrages français au Canada dont les textes et les gravures ont contribué, tout comme le rapprochement politique franco-britannique, "à façonner une image renouvelée de la France" vont, selon Gilles Gallichan, conforter la position des francophones au Canada et "ouvrir la voie à une reprise officielle des liens économiques et politiques entre le Canada et la France ${ }^{7}, w$

Ces vents favorables permirent à La Capricieuse de hisser ses voiles. Les textes de Robert Pichette ${ }^{8}$, Jean-François de Raymond ${ }^{9}$ et Jacques Portes $^{10}$ font, chacun à leur façon, la genèse et l'analyse des suites et séquelles de la mission de Belvèze sur les rives du Saint-Laurent, décrivant les éléments favorables du contexte géopolitique, les doutes et les hésitations des uns et

$$
++*
$$

6. Yvan LAMONDE, "L'exil dans l'exil: Le sejour de Louis-Joseph Papineau à Paris (1839-1845)", p. 176. Yvan LAMONDE et Didier POTON [ed.], La Capricieuse (I855): poupe et proue. Les relations France-Québec (1760-1914), Québec, Presses de l'Université Laval, 2006.

7. Gilles GALLICHAN, "La page avant la voile. Le livre et l'imprimé dans les relations France-Québec (1840-1855).", p. 189. Yvan LAMONDE er Didjer PơTON [éd.], La Caprncteuse (1855): poupe et proue. Les relatrons France-Quebec (17601914), Québec, Presses de l'Université Laval, 2006.

8. Robert PICHETTE, «La Capricieuse : elément d'une politique étrangère ou personnelle de Napoléon Ill à l'égard du Canada ? ", p. 191-209. Yvan LAMONDE et Didier POTON [éd.], La Capricieuse (I855): poupe et proue. Les relations France-Québec (1760-1914), Québec, Presses de l'Université Laval, 2006.

9. Jean-François DE RAYMOND, «La Capricieuse dans les archives françaises. L'initiative ex la décısion *, p. 21 1-226.

10. Jacques PORTES, "La Capricieuse (1855). Entre France, Grande-Breragne et Louisianes, p. 227-232. 
des autres, l'extrême prudence du commandant de La Capricieuse dans chacune de ses déclarations, l'accueil enthousiaste de la population et l'absence de réactions négatives de la part des autorités britanniques. La mission de La Capricieuse fut, au bout du compte, une affaire essentiellement canadienne; elle aura des suites commerciales et diplomatiques, notamment par l'instauration d'un consulat général à Québec, mais restera sans véritables lendemains. C'est pourtant justement alors que commence l'histoire de $\mathrm{La}$ Capricieuse, comme "événement considérable sur le plan symbolique et celui des relations globales entre le Canada et la France ${ }^{11}$."

Patrice Groulx ${ }^{12}$ et Yolande Grisé ${ }^{13}$ vont plonger, dans deux textes remarquables, au cour du symbolisme de l'événement, le premier l'étudiant tel qu'il apparaît en 1855, dévoilant la trace qu'en gardera la «mémoire collective" et sa signification identitaire, la seconde faisant un inventaire rigoureux de son inscription dans les fondements de la littérature nationale. Car il ne faut pas s'y tromper, si la venue de La Capricieuse, pour reprendre la formule de Françoise Lejeune, est une "fête de famille dans l'histoire des Canadiens" et un "non-événement dans l'histoire des Français ${ }^{14}$ ", il n'en demeure pas moins "un lieu commun de mémoire", essentiel à la compréhension du Canada français, puis du Québec.

L'analyse du discours qui se développe autour de cet événement montre bien la naissance et la genèse d'un mythe fondateur, au même titre que l'Histoire du Canada de Garneau ou le discours du balcon du général de Gaulle plus d'un siècle plus tard. C'est autour de cet événement que les textes des orateurs officiels, des commentateurs et des poètes vont cristalliser l'origine de l'affirmation identitaire. Les appels patriotiques, les évocations de l'histoire commune, la récurrence des métaphores familiales dans les discours, les drapeaux, les fêtes, tout cet apparat conduit Patrice Groulx à conclure que ce type de voyage, en apparence sans lendemain, s'est révélé " un lieu de mémoire complexe ${ }^{15}$ ". Les vers célèbres de Crémazie et les chansons publiées par Jacques-Adolphe Marsais, tout comme la centaine de milliers de mots ${ }^{16}$ publiés dans les neuf journaux de langue française en

$$
+4
$$

11. Jean-François DE RAYMOND, op. at., p 226.

12. Patrice GroUlX, "La Caprtcteuse en 1855: célébration et sugntication", p. 233-258. Yvan LAMONDE et Didier POTON [éd.], La Caprecieuse (1855): poupe et proute. Les relations France-Quebec (1760-1914), Québec, Presses de l'Université Laval, 2006.

13. Yolande GruSE, "Poétique de La Capricteusen, p. 259-284. Yvan LAMONDE et Didier POTON [éd.], La Capricieuse (1855): poupe et prouce. Les relatzons France-Québec (1760-1914), Québec, Presses de l'Université Laval, 2006.

14. Francoise LEJEUNE, op. ctt., p. 101.

15. Patrice GroulX, op. cit., p. 256.

t6. Yolande GrISE, op. cit., p. 262. 
activité au Canada entre le 13 juillet et le 25 août 1855, appartiennent autant à l'histoire que les rachitiques résultats économiques et l'absence quasitotale de rapprochement politique au cours des décennies qui vont suivre.

La dernière partie de l'ouvrage est davantage consacrée à la présentation et à l'analyse de certaines manifestations de l'intérêt de la France et au rôle de certains réseaux dans le développement des liens entre les deux peuples. Les textes sont ici plus disparates et, pour intéressants qu'ils soient, pas toujours reliés de façon directe à l'événement mémoriel central que constitue le voyage de $\mathrm{La}$ Capricieuse. Même si l'on ignore dans quelle mesure il est à l'origine de l'intérêt pour la colonisation française d'un Rameau de St-Père, cet intellectuel français n'en sera pas moins l'inspirateur, tant en Acadie qu'au Bas-Canada et en France, d'un réseau d'" Amis du Canada" qui s'inspireront de ses écrits et pousseront des Français à émigrer et les Canadiens à poursuivre leur œuvre colonisatrice. Pierre Trépanier note avec justesse que l'influence de Rameau s'exerce plus au Canada où ses relations se situent aux plus hauts niveaux politique et religieux qu'en France, où ses amis et lui ont du mal à se faire reconnaître en dehors des milieux religieux, des cercles d'érudits et des sociétés savantes ${ }^{17}$. C'est dans les milieux intéressés à l'expansion coloniale de la France ou aux sciences sociales naissantes, particulièrement chez les membres du mouvement leplaysien, que Rameau plaide pour la colonisation française du Canada et l'expansion, à travers tout le Canada et l'Acadie, des franco-catholiques. Avec ses amis intellectuels venus d'horizons idéologiques très différents - on songe ici au juriste catholique Claudio Jannet et au géographe protestant Onésime Reclus - il va tenter, pendant près de quarante ans, de fournir aux Canadiens les bases idéologiques pouvant conforter leur résistance à la modernité américaine et encourager la colonisation des terres encore vierges du nord de l'Ontario et des plaines de l'Ouest. Auteur de nombreux ouvrages et articles sur l'œuvre et l'action de Rameau de St-Pierre, Trépanier réussit une synthèse magistrale du parcours de ce personnage central de la seconde moitié du XIX siècle québécois.

Le tableau ${ }^{18}$ que dresse Guy Laperrière de la venue et la présence des communautés religieuses françaises au Canada démontre claịrement la

$$
+4
$$

17. Pierre Trépanier, "Rameau de St-Père, la France et la vie incellectuelle en Amérique française", p. 292. Yvan LAMONDE et Didier POTON [éd.], La Capncieuse (1855): poupe et proue. Les relations France-Québec (1760-1914), Québec, Presses de l'Université Laval, 2006.

18. Guy LAPERRIÈre, "Les communautés relıgieuses françaises au Québec (1792-1914) », p. 307-324. Yvan LAMONDE er Didier Poton [ed.], La Capricicuse (1855): poupe es proue. Les relations France-Québec (1760-1914), Québec, Presses de P'Université Laval, 2006. 
quasi-autonomie de ce phénomène par rapport à l'évolution des autres aspects des relations franco-canadiennes. Liée directement à la Révolution française, l'arrivée d'une première vague de religieux français réfractaires va avoir, tant par son nombre que par sa qualité intellectuelle, une grande influence sur le développement de l'Église canadienne et jouer un rôle déterminant au Bas-Canada. La seconde vague est issue des efforts incessants de l'évêque de Montréal, Mgr Bourget, pour attirer des communautés religieuses d'hommes et de femmes de France afin d'établir, au lendemain des rebellions libérales de 1837-1838, une société catholique au Canada. Et, évidemment, la troisième nous vient des lois républicaines de séparation de l'Église et de l'État, des mesures prises contre les congrégations religieuses, notamment l'expulsion des Jésuites et des autres congrégations non autorisées, jusqu'aux lois Combes au début du XXe siècle en passant par la laïcisation des structures scolaires sous Jules Ferry (1886). Seule la conclusion de cette excellente synthèse, qui affirme la faible influence des congrégations religieuses françaises au Canada au moment de La Capricieuse, laisse quelque peu songeur: que les congrégations amenées par Bourget une dizaine d'années auparavant se soient "canadianisées" ne diminue en rien, nous semblet-il, l'importance capitale qu'elles ont exercée sur l'ensemble du territoire.

Le dernier texte à présenter un réseau et ses figures de proue est celui de Gérard Fabre, qui s'attache à dépeindre, à travers la relation privilégiée unissant le premier ministre Wilfrid Laurier et le spécialiste de science politique André Siegfried, un réseau très actif, tissé cette fois entre "libéraux" des deux côtés de l'Atlantique. Souvent négligé au profit des chantres de l'idéologie conservatrice dominante, il s'agit néanmoins d'un réseau important qui, de Papineau à Honoré Beaugrand, de J.-G. Barthe à L.-O. David, de Dessaulles à Dandurand, a tissé des liens avec de nombreux et très influents penseurs, journalistes, hommes politiques et écrivains français. Cela ne se fait pas toujours sans quelque ambiguïté, les lignes de partage idéologique n'étant pas toujours les mêmes et les sujets de malentendus toujours présents, mais, Fabre nous le rappelle avec raison, même si l'anglomanie de Siegfried et son admiration pour Laurier l'amènent à sous-esțimer la montée en puissance du mouvement nationaliste, "les relations entre les deux pays se dirigent inéluctablement vers un état de plus grande égalité et annoncent celles que les Français vont entretenir désormais avec les Québécois ${ }^{19}$."

$$
+\div
$$

19. Gérard FaBre, "Un Français "conquis" : André Siegfried face à Wilfrid Laurier”, p. 360. Yvan LAMONDE et Dıdier Pơron [éd.], La Capricieuse (1855) - poupe et proue. Les relations France-Quebec (1760-1914), Québec, Presses de l'Université Laval, 2006. 
Le Commissaire de la Marine, Jean-Marie Huillé, nous livre un compte rendu et un sympathique témoignage de la mission de la frégate française L'Aventure de l'escadre de Terre-Neuve, venue célébrer à Québec, en 1955, le centième anniversaire de la venue de La Capricieuse. Cérémonies militaires, sociales et religieuses marquèrent de façon toute modeste, mais dans un cadre chaleureux, un événement auquel personne dans le Québec "duplessiste" ne donnait la signification qu'il retrouvera avec la Révolution tranquille et la reprise des liens directs entre la France et le Québec.

L'étude que propose Patrick Cabanel ${ }^{20}$ de la place du Canada dans les lectures scolaires de la Troisième République ne manque pas d'intérêt, même s'il laisse le lecteur sur sa faim. En effet, comment arriver à détacher ce type d'ouvrages des centaines de livres et des milliers d'articles publiés en France à la même époque sur le Canada, et dont plusieurs étaient remis en prix de fin d'année aux élèves méritants? Cabanel rappelle évidemment l'importance et la signification du Tour de France par deux enfants de G. Bruno dont on connaît le succès fulgurant et constant après 1877 . Si la France a perdu ses deux provinces d'Alsace et de Lorraine, elle demeure un grand pays à la géographie diversifiée que les deux orphelins vont explorer. Le phénomène du "tour de la nation par des enfants" a eu un tel succès qu'il est très intéressant d'y découvrir la place qu'y occupe le Canada. Ainsi les "provinces perdues" ne sont-elles pas oubliées mais en attendant leur reconquête, c'est à la construction d'un puissant sentiment national que sont conviés les enfants de France. Si le succès du livre amène d'autres auteurs à utiliser le même topos littéraire pour l'Algérie et les autres colonies françaises, cinq au moins "ont choisi d'insérer une étape canadienne dans un tour de la France Outre-mer" et, dans le cas de Georges Lamy, de consacrer au Canada un ouvrage entier ${ }^{21}$. En plus d'une leçon d'histoire et de géographie, ces livres entretiennent soigneusement la nostalgie d'une colonisation française qui a réussi malgré l'échec militaire et surtout, constitue une façon indirecte mais très éloquente de parler sans les nommer, par un effet de déplacement, des provinces perdues et de la grandeur de la France: le Québec retrouvé c'est un peu la contrepartie des provinces détachées du territoire national en 1870.

Cherchant à mieux comprendre l'importance et la signification de la mission de La Capricieuse, en l'éclairant à l'en amont par une analyse des

$$
+4
$$

20. Parrick CABAnel, aLautre province perdue: le Canada, dans les livres de lecture scolaire sous la Troisì̀me Républiquew, p. 328-341. Yvan LAMONDE et Didier POTON [éd.], La Capricieuse (1855): poupe et proue. Les relations France-Québec (1760-1914), Québec, Presses de l'Université Laval, 2006.

21. Georges LAMY, Le voyage du novice Jean-Paul d̀ travers la France d'Amerque, Paris, Collin, 1890, p. 29. 
relations France-Bas-Canada avant 1855 et à bien décrire l'importance et surtout les limites de l'événement lui-même, les organisateurs du colloque et les participants ont fortement contribué à la compréhension de ce que le président québécois de la Commission franco-québécoise sur les lieux communs de mémoire, Marcel Masse, a appelé de ses vœux, soit une approche qui tienne compte des aspects historiques, ethnologiques, sociologiques, scientifiques et artistiques ${ }^{22}$. Tout en regrettant et en comprenant la portion congrue réservée à l'aval de l'événement, sans doute beaucoup trop riche pour faire l'objet d'autre chose que de coups de sonde bien choisis, il faut ici rendre hommage aux éditeurs et aux organisateurs du colloque, pour ce livre magnifique qui nous entraîne dans un voyage riche et passionnant aux sources d'une identité toujours en construction.

Sylvain Simard

Député de Richelieu

Président de la Commission de la culture

Assemblée nationale du Québec

\section{Denise Deshaies et Diane Vincent [éd.] \\ Discours et constructions identitaires \\ Québec, Presses de l'Université Laval, 2004.}

Composé autour d'une réflexion entamée lors du séminaire "Discours et constructions identitaires" (la discipline, le niveau et les activités du séminaire menant à la rédaction du collectif ne sont pas expliqués), l'ouvrage dirigé par Denise Deshaies et Diane Vincent, regroupant des contributions de chercheurs chevronnés et d'étudiants, a pour objectif de circonscrire diverses facettes de la construction identitaire par le biais de discours (surtout sur un "nous français" au Canada (p. XI)) :

Si la langue constitue pour plusieurs communautés un symbole qui permet aux individus de se définir et de définir leur rapport aux autres, elle est également l'outil par lequel se construit l'identité [...]. Les collaborateurs à cet ouvrage souscrivent à une telle position, abordant la question identitaire à travers les marques linguistiques qui façonnent les représentations. (p. X; je souligne)

$$
+\div+
$$

22. Marcel MASSE, "Mot de bienvenue», p. VIII. 